\title{
METABOLIC AND ADIPOCYTOKINE PRECONDITIONS FOR PROGRESSION OF NON-ALCOHOLIC STEATOHEPATITIS IN OBESITY PATIENTS DUE TO COMORBIDITY WITH CHRONIC OBSTRUCTIVE PULMONARY DISEASE
}

\author{
Olha Hryniuk $^{1}$, Oksana Khukhlina ${ }^{2}$, Oksana Liakhovych ${ }^{3}$, \\ Viktoriia Hutsuliak $^{4}$, Snizhana Hnatkovych ${ }^{5}$
}

\begin{abstract}
:
The increasing prevalence of chronic obstructive pulmonary disease (COPD) and nonalcoholic steatohepatitis (NASH) is a problem of high importance.

Objective: to determine the state of blood lipid spectrum, glycemia, the degree of insulin resistance and their regulation by adipose tissue hormones in NASH patients against the background of obesity, depending on comorbidity with COPD.

Methods. 130 patients were examined, including 35 NASH patients with obesity of the 1st stage (1 group), 60 NASH patients with obesity of the 1st stage and COPD 2-3 D (group 2), and 35 patients with COPD 2-3 D (group 3).

Results: Blood lipid concentration in patients of the 1st and the 2nd groups exceeded the norm by $29.5 \%$ and $39.8 \%$ respectively $(\mathrm{p}<0.05)$. In the 3rd group - by $14.9 \%(\mathrm{p}<0.05)$. The content of total cholesterol in the blood also points out its probable increase by $36.3 \%, 45.7 \%$ and $14.9 \%(\mathrm{p}<0.05)$ in comparison with practically healthy individuals (PHI) in patients of the 1st, 2nd and 3rd groups. A probable increase in the concentration of triacylglycerols (TG) in blood (1.9 and 2.2 times, respectively $(\mathrm{p}<0.05)$ ) was recorded in the 1st and 2nd groups of patients, while in patients of the 3rd group the changes were quite significant (1.6 times increase, $\mathrm{p}<0.05$ ).

Conclusions. Comorbidity of COPD in obese patients and NASH is an additional, powerful-inducing factor of lipid distress syndrome with significantly higher increase (compared with NASH without lung pathology) TG in blood, which form the basis of liver steatosis, total cholesterol, low density lipoproteins, with significantly higher decrease high density lipoproteins, the atherogenic index, which are accompanied by hyperleptinemia, adiponectin deficiency, correlate with the degree of liver steatosis, fibrosis index, cytolytic activity, cholestatic, mesenchymal-inflammatory syndromes and are interrelated with hyperleptinemia, hypoadiponectinemia.
\end{abstract}

UDC Classification: 616.36, DOI: https://doi.org/10.12955/pmp.v1.94

Keywords: nonalcoholic steatohepatitis, chronic obstructive pulmonary disease, obesity

\section{Introduction}

The prevalence of non-alcoholic steatohepatitis (NASH) is gaining global significance in the population of economically developed countries with a growing tendency in Ukraine. According to various authors, the incidence of NASH ranges from $20 \%$ to $35 \%$, which directly affects the quality of life of patients, contributes to the progression of disorders of all types of metabolism and development of hepatic cell insufficiency (Viglino et al., 2017).

NASH is known to be a progressive form of non-alcoholic fatty liver disease (NAFLD) and is characterized by increased activity of liver enzymes in the blood and morphological changes of the organ, developing against the ground of steatosis and inflammation, balloon dystrophy of hepatocytes, centrolobular necrosis, steatosis (Lazo et al., 2013).

It is important to note that a close pathogenic association of NAFLD with obesity, insulin resistance (IR), hypertension and dyslipidemia enables us to consider non-alcoholic fatty dystrophy as hepatic manifestation of metabolic syndrome (MS) (Dowman, 2011). Intra-abdominal adipocytes of the adipose tissue have a high density of beta-adrenergic receptors, corticosteroid and androgen receptors, and a relatively lower density of alpha-2-adrenergic receptors and insulin receptors. This determines a high sensitivity of the visceral adipose tissue to the lipolytic action of catecholamines and low sensitivity to the anti-lipolytic action of insulin (Anstee et al. 2013). Intense lipolysis in the intra-abdominal adipocytes results in the release of a large number of free fatty acids (FFA) entering the liver through the portal vein and then into the systemic circulation. Thus, the liver is exposed to a powerful and

\footnotetext{
${ }^{1}$ HSEI of Ukraine "Bukovinian State Medical University", Chernivtsi, Ukraine, Department of Internal Medicine, Clinical Pharmacology and Occupational Diseases, hryniuk.olha@gmail.com

${ }^{2}$ HSEI of Ukraine "Bukovinian State Medical University", Chernivtsi, Ukraine, Department of Internal Medicine, Clinical Pharmacology and Occupational Diseases, oksanakhukhlina@gmail.com

${ }^{3}$ HSEI of Ukraine "Bukovinian State Medical University", Chernivtsi, Ukraine, Department of Internal Medicine, Clinical Pharmacology and Occupational Diseases, ksiusuk@gmail.com

${ }^{4}$ HSEI of Ukraine "Bukovinian State Medical University", Chernivtsi, Ukraine, hutsuliak@ukr.net

${ }^{5}$ HSEI of Ukraine "Bukovinian State Medical University", Chernivtsi, Ukraine, snizhana.gn@ukr.net
} 
constant impact of FFA, which leads to a number of metabolic disorders with the development of insulin resistance (IR) and systemic hyperinsulinemia (Mykhailovska, Miniailenko, 2016).

After establishing the endocrinological activity of the adipose tissue, the main positions of the hypothalamic-pituitary theory and the role of leptin and adiponectin, the lipocentric theory was transformed into adipokine, according to which the basis for the formation of dysmetabolic disorders, components of metabolic syndrome (MS) which are burdened by "faulty" links of the pathological process, is the effect of signal molecules produced by lipocytes but not changes of in the energy substrate of adipocyte restructuring (Radchenko, Pylypiv, Zukow, 2017). Many studies have proved the role of leptin and adiponectin in exacerbation of COPD and in its stable period (Turer, Scherer, 2012), (Baltieri et al., 2018).

Recently, John G. Elliot and co-authors (2019) have suggested an additional mechanism whereby impaired respiratory function in obesity is associated with accumulation of the adipose tissue in the respiratory tract. Adipocyte accumulation can contribute to bronchial remodeling and bronchial obstruction. Some sources report that non-alcoholic fatty liver disease is widespread in chronic obstructive pulmonary disease (Jung et al., 2012, Viglino et al., 2017).

The mechanisms underlying NASH and pulmonary dysfunction in patients with COPD against the ground of obesity remain unclear, which necessitates the research dealing with the links of burden and search for new factors in pathogenesis of progression of this comorbid pathology.

\section{Aim}

The objective of the study was to determine the state of blood lipid spectrum, glycemia, the degree of insulin resistance and their regulation of adipose tissue hormones in patients with NASH against the ground of obesity, depending on the comorbidity of COPD.

\section{Material and Methods of Investigation}

130 patients were examined, of which 35 were patients with NASH and obesity grade 1 (group 1), 60 patients with NASH and obesity grade 1 and COPD 2-3 D (group 2) and 35 patients with COPD 2-3 D (group 3). The average age of the patients was $(55.7 \pm 3.22)$ years. The control group consisted of 30 practically healthy individuals (PHIs).

The diagnosis of NASH was made according to the unified clinical protocol approved by the order of the Ministry of Health of Ukraine No. 826 of 06.11.2014, in the presence of criteria for the exclusion of chronic diffuse liver disease of viral, hereditary, autoimmune or drug genesis as the cause of cytolytic, cholestatic, mesenchymal-inflammatory syndromes, and the results of ultrasonography (USG) with shear wave elastography, steatotest.

The diagnosis was made and treatment of COPD conducted in accordance with the recommendations of clinical guidelines (MOH Order No. 555 of June 27, 2013, taking into account the recommendations of GOLD, 2019).

The diagnosis of obesity was made according to the classification of the WHO International Obesity Working Group (1997). Patients were subjected to measurements of height and body weight, their body mass index (BMI) was calculated according to the Kettle formula (1):

$\mathrm{BMI}=\operatorname{mass}(\mathrm{kg}) /$ height $^{2}(\mathrm{~m})(1)$

The diagnosis of obesity was made with BMI higher than $30 \mathrm{~kg} / \mathrm{m}^{2}$.

On admission of patients to the hospital, the functional state of the liver was determined according to the approved list of activity of enzymes, markers of pigment and nitrogen metabolism, proteinogram, lipidogram, ionogram, and calculation of the de Ritis coefficient. All the patients underwent anthropometry to determine body mass index (BMI), waist circumference (WC), hip circumference $(\mathrm{HC})$, and waist / hip index (WHI = WC / HC).

The degree of liver steatosis and its nature was determined using the ratified kit SteatoTest, ASH and NASH-Test (BioPredictive, France). The stage of liver fibrosis was determined by using a ratified set of markers for the quantitative biochemical assessment of fibrosis "FibroTest" (BioPredictive, France).

Carbohydrate metabolism was determined by fasting glycemia level and 2 hours after glucose loading (glucose tolerance test) by glucose oxidase method, fasting insulin (DRG System) by enzyme-linked immunosorbent assay (ELISA), blood glycosylated hemoglobin (HbA1c) content using standard Simko 
Ltd reagent kits (Lviv, Ukraine) by the VA Koroleva method. The degree of IP was determined by the body mass index (BMI), WC / HC; HOMA-IR index (Matthews et al., 2008), which was calculated using the HOMA Calculator Version 2.2 Diabetes Trials Unit of the University of Oxford (United Kingdom).

Blood lipid spectrum was studied for total lipid content, total cholesterol (TC), triacylglycerols (TG), low density lipoprotein (LDL) and high density (HDL) cholesterol using Diagnostic standard kits of Simko Ltd. The atherogenicity index (IA) (2) was calculated:

$\mathrm{IA}=\mathrm{TC}-\mathrm{HDL} / \mathrm{HDL}$

Hormonal regulation of lipid metabolism was assessed by blood leptin, adiponectin (DRG System) by ELISA.

Statistical analysis of the results was performed according to the type of study conducted and the types of numerical data that were obtained. The normality of the distribution was checked using the Lilliefors, Shapiro-Wilk tests and the method of direct visual evaluation of the histograms of the distribution of eigenvalues. Quantitative indicators that had a normal distribution are presented as mean $(\mathrm{M}) \pm$ standard deviation (S). In the nonparametric distribution, the data are presented in the form of a median (Me) as a measure of position, upper $(\mathrm{Q} 75)$ and lower quartiles $(\mathrm{Q} 25)$ as a scatter measure. Discrete values are presented as absolute and relative frequencies (percentage of observations to the total number of surveyed). For the comparisons of data that had a normal distribution, parametric tests were used, particularly, Student's t-test, Fisher's F-test. In case of abnormal distribution, the following was used: median test, calculation of the rank Mann-Whitney U-test, for a multiple comparison Wilcoxon T-test was applied (in case of dependent groups). Pearson correlation analysis for parametric distribution and Spearman's rank correlation coefficient were used to estimate the degree of dependence between variables in case of the distribution of indicators that were significantly different from normal. For statistical and graphical analysis of the obtained results Statistic for Windows software version 8.0 (Stat Soft Inc., USA), Microsoft Excel 2007 (Microsoft, USA) were used.

\section{Results of the Investigation}

The study of the blood lipid spectrum in patients with NASH showed a number of similar changes (Table 1 ), however, certain differences in the degree of probability depending on the presence of COPD comorbidity were found.

Thus, the total blood lipid concentration in patients of the 1st and 2nd groups exceeded the norm by $29.5 \%$ and $39.8 \%(\mathrm{p}<0.05)$, respectively, and in the 3rd group - the lowest of the comparison groups by $14.9 \%(\mathrm{p}<0.05)$ with a significant statistical difference between the groups $(\mathrm{p}<0.05)$. Blood content of total cholesterol also indicates its likely increase by $36.3 \%$ and $45.7 \%$ ( $\mathrm{p}<0.05)$ compared with PHI with patients of the 1st and 2nd groups, as well as a probable excess of the indicator in PHI and patients of group 3 (by $14.9 \%(p<0.05)$ ). A probable increase in TG blood concentration (1.9 and 2.2 times, respectively $(\mathrm{p}<0.05))$ was registered in the 1 st and 2 nd groups of patients, while the changes in the 3rd group were significant as well $(1.6$-fold increase, $\mathrm{p}<0.05)$. That is, the blood TG content of NASH comorbidity with obesity and COPD was probably higher than in patients with NASH with obesity without COPD. In patients with COPD with normal body weight there was significant hypertriacylglycerolemia, which may be a contributing factor in the development of liver steatosis in future even in absence of obesity.

The study of blood concentrations of proatherogenic lipoprotein fractions indicates a number of probable changes: the LDL cholesterol concentration in patients of group 1 was significantly higher than the control indicator by 1.7 times $(\mathrm{p}<0.05)$, and in patients of 2 nd and 3 groups showed a probable increase in LDL cholesterol by 1.8 and 1.3 times $(\mathrm{p}<0.05)$, respectively, with a significant statistical difference between the groups $(p<0.05)$. It should be noted that the increase in the blood content of total cholesterol and proatherogenic LDL in COPD patients may be an important prognostic factor in the development of atherosclerosis in these patients. The blood concentration of antiatherogenic lipoproteins - HDL cholesterol in patients in group 1 of observation was 1.4 times lower $(\mathrm{p}<0.05)$ compared to that of the control (Table 1). At the same time, in patients of groups 2 and 3 - the indicator was not expected to increase by 1.6 and 1.2 times, respectively, compared to the indicator in PHI ( $<<0.05)$. As can be seen from the results of the study, patients with NASH without COPD have a deficiency of HDL, which indicates a minimum level of protection of the vascular endothelium against aggression by free radicals 
and atherogenic fractions of blood lipids, a high risk of atherogenesis. Moreover, patients with COPD and NASH with COPD have hyperproduction of this fraction of lipoproteins, although the source is not completely known. Probably, with the COPD comorbidity in the exacerbation phase, some fats are evacuated from the pulmonary cells that enter the bloodstream and enter the liver, where they are deposited due to the blocked $\beta$-oxidation of fatty acids.

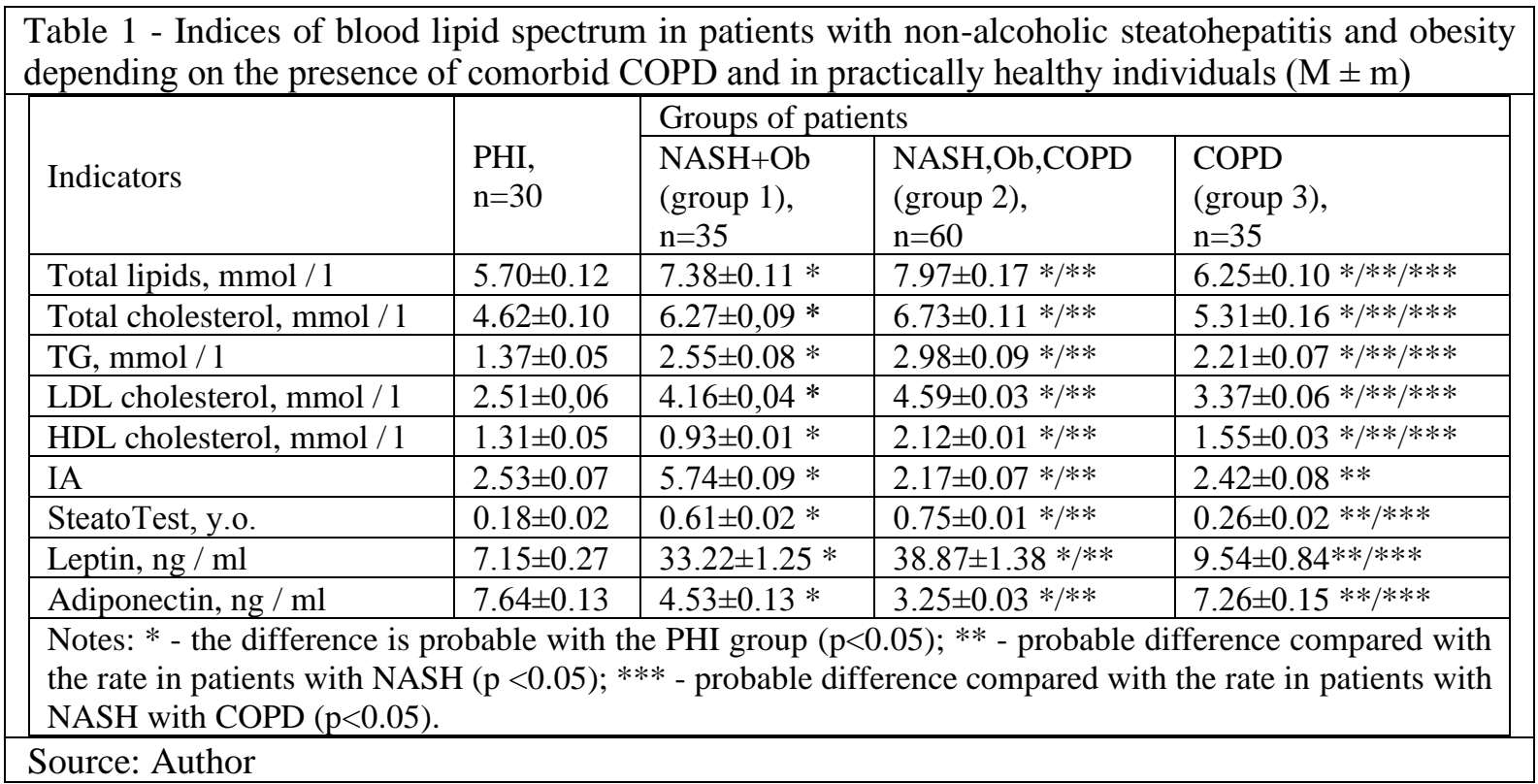

These changes resulted in a significant increase in the atherogenic index (IA) in patients of the 1st group - 2.3 times, and in the 2nd group the indicator was lower than in the PHI - 1.2 times $(\mathrm{p}<0.05)$, in patients of group 3 - changes were not significant $(p>0.05)$ (table. 1). That is, maximal changes of IA are found in patients with NASH and obesity, which indicates, on the one hand, the presence of significant risk factors for the progression of atherosclerosis in these patients, and on the other, an extremely unfavorable pathogenetic situation for the development and progression of NASH in COPD patients. At the same time, the risk of atherogenesis in COPD patients and NASH with COPD is likely to be lower, but the risk of progression of lipid distress syndrome, obesity, and degree of liver steatosis increases. The evidence of the correctness of our hypothesis is the change in steatotest, which showed a significantly increased index of steatosis (IP) of hepatocytes in patients with NASH with obesity (3.4 times $(p<0.05)$ ), while in patients with comorbid NASH and COPD excess was 4.2-fold ( $<<0.05)$ (see Table 1). It should be noted that the rates of steatotest in patients with COPD with normal body weight were not statistically different from the indicator in PHI, but were at the upper limit of normal ( $p>0.05)$.

Thus, the most unfavorable lipid spectrum among the comparison groups concerning the content of atherogenic fractions of lipoproteins and TG, which are the basis of liver steatosis, was established in patients with NASH with accompanying COPD and obesity, which is further enhanced by a significant increase in the content of fractions of HDL, which increases the risk of liver steatosis by 1.2-fold $(\mathrm{p}<0.05)$.

The analysis of blood content of adipocytokines of leptin and adiponectin depended on the number of comorbid conditions. Thus, the leptin content of blood in patients of group 1 exceeded the indicator in PHI by 4.7 times, and in patients of group $2-5.4$ times with the presence of intergroup difference $(\mathrm{p}<0.05)$. The adiponectin blood content of patients in group 1 was 1.7 times lower than that in PHI, and 2.4 times in patients in group 2, with the presence of intergroup difference $(\mathrm{p}<0.05)$ (Table 1). Patients in 3 groups the changes of indicators were not established.

The results of glycemia, insulinemia and IR indices in patients with NASH with isolated and comorbid COPD and obesity are presented in Table 2 . Analysis of the studies showed that patients in the 1st and 2nd groups showed a slight likelihood of an increase in fasting glycemia by $11.4 \%$ and $14.7 \%(\mathrm{p}<0.05)$ compared to the control group, while in patients of group 3 changes in glycemia were unlikely (Table 2). Analysis of the postprandial glycemia indices obtained during GTT in patients of the 1st and 2nd groups also showed an increase in glucose content after $120 \mathrm{~min}$ after loading - by $18.8 \%$ and $34.6 \%$ 
$(\mathrm{p}<0.05)$, respectively, compared to those in the PHI group, while in group 3 changes were unlikely ( $>0.05)$. The fasting insulin study found probable hyperinsulinemia, which in patients of group 1 exceeded the indicator in the PHI group by 2.4 times, in patients in group 2, the fasting insulin content exceeded the norm by 2.9 times $\left(\mathrm{p}_{1-2}<0.05\right.$ ) (see Table 2 ). The presence of peripheral tissue sensitivity disorders to insulin in patients with NASH and obesity indicates a significant increase in the fasting HOMA IR index (in groups 1 and 2, respectively, by 2.4 and 2.9-fold $(\mathrm{p}<0.05)$ ) with a significant difference between these groups $(\mathrm{p}<0.05)$. Patients with NASH against the ground of obesity and COPD have been diagnosed with the highest manifestation of IR syndrome, which is likely to be primary (hereditary predisposition), and possibly secondary to liver damage due to steatosis.

\begin{tabular}{|c|c|c|c|c|}
\hline \multirow[b]{2}{*}{ Indicators } & \multicolumn{4}{|c|}{ Groups of patients } \\
\hline & $\begin{array}{l}\text { PHI, } \\
\mathrm{n}=30\end{array}$ & $\begin{array}{l}\mathrm{NASH}+\mathrm{Ob} \\
\text { (group } 1) \\
\mathrm{n}=35\end{array}$ & $\begin{array}{l}\text { NASH,Ob,COPD } \\
\text { (group 2), } \\
\text { n=60 }\end{array}$ & $\begin{array}{l}\mathrm{NASH}+\mathrm{Ob} \\
\text { (group 1), } \\
\mathrm{n}=35\end{array}$ \\
\hline Fasting glucose, $\mathrm{mmol} / \mathrm{l}$ & $5.12 \pm 0.10$ & $5.68 \pm 0.13 *$ & $5.85 \pm 0.11 * / * *$ & $5.27 \pm 0.09 * * / * * *$ \\
\hline Postprandial glucose, $\mathrm{mmol} / \mathrm{l}$ & $7.41 \pm 0.21$ & $8.79 \pm 0.27 *$ & $9.96 \pm 0.25 * / * *$ & $7.73 \pm 0.25 * * * * * *$ \\
\hline Insulin, mcOD / ml & $9.93 \pm 2.11$ & $23.35 \pm 2.38 *$ & $28.52 \pm 2.26 * / * *$ & $15.85 \pm 2.14 * * / * * *$ \\
\hline $\mathrm{Hb} \mathrm{A_{1C } , \%}$ & $5.05 \pm 0.12$ & $5.53 \pm 0.10 *$ & $5.67 \pm 0.11 * / * *$ & $5.09 \pm 0.15 * * / * * *$ \\
\hline HOMA-IR & $1.29 \pm 0.07$ & $3.04 \pm 0.12 *$ & $3.70 \pm 0.13 * / * *$ & $2.05 \pm 0.10 * / * * / * * *$ \\
\hline \multicolumn{5}{|c|}{$\begin{array}{l}\text { Notes: } * \text { - the difference is probable with the PHI group }(\mathrm{p}<0.05) ; * * \text { - probable difference compared with } \\
\text { the rate in patients with NASH }(\mathrm{p}<0.05) ; * * * \text { - probable difference compared with the rate in patients with } \\
\text { NASH with COPD }(\mathrm{p}<0.05) \text {. }\end{array}$} \\
\hline
\end{tabular}

Table 3 - Matrix of correlation relationships between blood content of cholesterol, TG, major fractions of lipoproteins, glucose, IR and markers of cytolysis, cholestasis, mesenchymal inflammation, degree of steatosis, liver fibrosis, bl ood content of leptin, adiponectin $(r, p)$

\begin{tabular}{|l|l|l|l|l|l|l|l|l|}
\hline Indicator & Bilirubin & ALT & GGT & $\begin{array}{l}\text { Timol. } \\
\text { test }\end{array}$ & $\begin{array}{l}\text { Steato- } \\
\text { test }\end{array}$ & $\begin{array}{l}\text { Fibro- } \\
\text { test }\end{array}$ & Adiponectin & Leptin \\
\hline TC & $0.33^{*}$ & $0.37^{*}$ & $0.52^{*}$ & $0.34^{*}$ & $0.70^{*}$ & $0.55^{*}$ & $-0.53^{*}$ & $0.67^{*}$ \\
\hline TG & $0.51^{*}$ & $0.55^{*}$ & $0.63^{*}$ & $0.54^{*}$ & $0.79^{*}$ & $0.57^{*}$ & $-0.57^{*}$ & $0.72^{*}$ \\
\hline LDL & $0.36^{*}$ & $0.39^{*}$ & $0.56^{*}$ & $0.37 *$ & $0.75^{*}$ & $0.56^{*}$ & $-0.55^{*}$ & $0.68^{*}$ \\
\hline HDL & -0.15 & -0.22 & -0.26 & $-0.34^{*}$ & $-0.62^{*}$ & $-0.48^{*}$ & $0.59^{*}$ & $-0.53^{*}$ \\
\hline IA & 0.11 & 0.23 & $0.38^{*}$ & $0.30^{*}$ & $0.55^{*}$ & $0.39^{*}$ & $-0.43^{*}$ & $0.57^{*}$ \\
\hline Glucose & $0.38^{*}$ & $0.45^{*}$ & $0.38^{*}$ & $0.47^{*}$ & $0.59^{*}$ & $0.57^{*}$ & $-0.58^{*}$ & $0.69^{*}$ \\
\hline Insulin & $0.35^{*}$ & $0.42^{*}$ & $0.36^{*}$ & $0.45^{*}$ & $0.56^{*}$ & $0.52^{*}$ & $-0.56^{*}$ & $0.72^{*}$ \\
\hline $\begin{array}{l}\text { HOMA } \\
\text { IR }\end{array}$ & $0.41^{*}$ & $0.53^{*}$ & $0.47 *$ & $0.61 *$ & $0.73^{*}$ & $0.65^{*}$ & $-0.69^{*}$ & $0.78^{*}$ \\
\hline & & & & & & & \\
\hline
\end{tabular}

Source: Author

At the same time, in patients with normal body weight, the insulin content in the blood, although not significantly, exceeded the index in PHI 1.6 times ( $>>0.05$ ), which contributed to the probable increase of HOMA-IR 1.6 times $(\mathrm{p}<0.05)$. That is, the probable increase in the degree of IR in patients with COPD in the absence of obesity indicates a significant effect of COPD in pathogenesis of NASH progression in their comorbidity. The mechanism of IR development in patients with COPD will be the prospect of our further study.

Analysis of the results of the study of hemoglobin glycosylation degree showed a probable increase in a relative content of $\mathrm{HbA} 1 \mathrm{c}$ in patients of the 1st and 2nd groups, which exceeded the indicator in PHI by $10.6 \%$ and $13.4 \%(\mathrm{p}<0.05)$, indicating that the presence of episodes of latent hyperglycemia (Table 2 ) in this group of obese patients.

Binary correlation analysis between blood lipid spectrum, glycemia and insulin content with markers of biochemical syndromes of NASH and blood content of leptin and adiponectin (Table 3) indicates that the greatest influence on the development of NASH is produced by TG and LDL cholesterol, postprandial glucose and IR. 
In NASH patients with COPD, blood content of cholesterol, TG, LDL cholesterol and IA correlated directly with markers of such syndromes as cytolysis, cholestasis, mesenchymal inflammation, degree of steatosis and liver fibrosis, blood content of leptin and inversely dependent adiponectin $(\mathrm{p}<0.05)$ (Table 3).

A strong degree of correlation between NOMA IR and blood leptin and adiponectin content, markers of mesenchymal inflammation, degree of liver steatosis and fibrosis $(\mathrm{p}<0.05)$ was established (Table 3$)$. All investigated parameters are probable risk factors and direct links to pathogenesis of NASH progression against the ground of obesity and COPD.

\section{Discussion of Research Results.}

The analysis of the obtained results showed a probable increase in the level of postprandial glycemia in patients with NASH and COPD compared with NASH without COPD, and the blood glucose level increased with increasing levels of adipose tissue hormone - leptin $(\mathrm{p}<0.05)$. The level of glycosylated hemoglobin in the blood, as a marker of the duration of persistence and intensity of hyperglycemia, probably exceeded the PHI in both groups $(\mathrm{p}<0.05)$, which confirms the role of chronic postprandial hyperglycemia and enhanced glycosylation of transport proteins in the pathogenesis of NASH with underlying obesity, and is indicative of latent hyperglycemia and the realization of the phenomenon of glucose toxicity. At the same time, HbA1c was significantly higher in NASH patients with COPD than in NASH patients without COPD, which is probably due to the negative effects of hypoxia on carbohydrate metabolism and induction of the IR phenomenon. Confirmation of the increase in the degree of IR in NASH in comorbidity with COPD was an increase in blood insulin levels of 2.9 times vs 2.4 times - in patients with NASH $(\mathrm{p}<0.05)$. This fact indicates the role of COPD, namely hypoxia, in the development of functional insulin deficiency, or IR, and in general, in the progression of NASH with underlying obesity. Calculation and analysis of IR indices confirms the presence of significant peripheral tissue IR in patients with NASH, and the HOMA IR index increases significantly with comorbidity with COPD $(\mathrm{p}<0.05)$, which indicates that not only obesity and MS are the causes of IR, but also systemic hypoxia and systemic inflammation in COPD can inhibit the sensitivity of receptors to insulin.

Disorders of glycemic and the insulin profile of blood in patients with NASH partially depended on the degree of activity of cytolysis, cholestasis and mesenchymal inflammation in weak and moderate relationship, but the total effect of postprandial glycemia and hyperinsulinemia on the development of clinical and biochemical syndromes of NASH was found to be considerable: with the degree of hepatic steatosis, liver fibrosis index; and the degree of this relationship increased with comorbidity with COPD. This fact is also indicative of disorders in the processes of neutralization of insulin in the liver in patients with NASH, i.e. an increase in the intensity of the syndrome of hepatocellular insufficiency due to increased activity of cytolysis and necrosis of hepatocytes. The nature of the curve, which reflects the tendency of changes in patients with NASH and obesity, indicates the progression of disorders of insulin neutralization in comorbidity with COPD. An important reason for the increase in the degree of IR is the phenomenon of glycosylation of not only transport but also structural proteins of membranes, which also include insulin receptors. Changes in the conformational structure of receptor proteins due to enhanced glycosylation contribute to the desensitization of the latter to the effects of the hormone and the progression of tissue IR. The data obtained indicate that in patients with NASH, which developed against the ground of obesity, IR syndrome is primary and its presence is the basis for the development of NAFLD. The presence of hypoxia and respiratory acidosis in comorbid COPD contributed to increased IR in NASH and obesity, increased glycosylation of hemoglobin, progression of metabolic disorders, an increased degree of steatosis and fibrosis of liver tissue. Thus, in obese patients with $\mathrm{NASH}$, the preconditions for the development of nonalcoholic fatty liver disease are probable postprandial hyperglycemia, increased glycosylation of transport proteins, compensatory hyperinsulinemia, increased peripheral tissues IR, which are exacerbated by comorbid COPD. Disorders of carbohydrate and lipid metabolism under IR conditions are a cardinal metabolic predictor for the development of NAFLD in comorbidity with COPD also due to the implementation of the phenomena of glucose and lipotoxicity, which assume the accumulation of non-metabolized products of lipid catabolism and anaerobic glycolysis, average mass molecules in the systemic circulation, hyperketonemia, systemic metabolic and respiratory acidosis. 
The most metabolically significant precondition for the development of NASH, which is causally associated with IR syndrome, is persistent lipid distress syndrome and disorders of lipid metabolism in general. The analysis of blood lipid spectrum studies showed that in patients with NASH without COPD there is an increase in the level of total cholesterol in the blood by 1.4 times $(\mathrm{p}<0.05)$, in patients with NASH with COPD was registered its probable increase by 1.5 times $(\mathrm{p}<0.05)$ in comparison with PHI. At the same time there was a slight but probable excess of blood cholesterol in patients with COPD with an isolated course $(\mathrm{p}<0.05)$, which indicates the involvement of inflammatory bronchial disease in disorders of lipid metabolism. Confirmation of our hypothesis was a probable increase in the concentration of TG in patients with NASH with COPD in 2.2 times against 1.9 in NASH $(p<0.05)$, while in patients with isolated COPD with normal body weight the changes were quite significant unexpectedly - an increase in TG content by 1.6 times $(\mathrm{p}<0.05)$. Considering the fact that the pathomorphological basis of hepatic steatosis is accumulation of neutral fat in hepatocytes in the form of TG, COPD against the ground of NASH and obesity significantly contributes to this process. Activity of the endothelial enzyme lipoprotein lipase is proved to decrease under conditions of hypoxia, acidosis and oxidative stress, which leads to a decrease in the intensity of TG hydrolysis and prolonged blood circulation of chylomicrons, and very low density lipoproteins that are overloaded with TG. Considering the fact that the leading consequences of metabolic disorders in COPD are tissue hypoxia, oxidative and nitrosive stress, acidosis and energy starvation, the effect of this comorbidity on the course of NASH becomes clear.

The study of blood concentrations of proatherogenic fractions of lipoproteins indicates that the concentration of LDL cholesterol in patients with NASH and COPD was significantly higher than in PHI 1.8 times $(p<0.05)$, and in patients with NASH without COPD in 1.7 times $(p<0.05)$, while in patients with isolated COPD with normal body weight there was a 1.3-fold increase in LDL cholesterol $(\mathrm{p}<0.05)$. This fact with this comorbidity indicates a causal relationship between the increase in the degree of hyperlipidemia and hepatic steatosis and comorbidity with COPD. Disturbance of $\beta$-oxidation of FFA and lipogenesis under IR conditions, as well as enhanced lipolysis of deposited lipids in visceral fat depots lead to increased supply of non-esterified FFA to the liver in order to be used as energy material, TG biosynthesis, formation of LDL compounds with glycerol and storage in the form of neutral fats in the cytoplasm of hepatocytes. A direct, high-strength correlation between the indicators of TG content in the blood and the degree of hepatocyte steatosis indicates a strong interdependence of these processes. Lipid distress syndrome due to the comorbidity of NASH with COPD is characterized by a significant increase of HDL cholesterol content in the blood, while in patients with NASH it decreased with a significant increase in the atherogenic index $(\mathrm{p}<0.05)$. Although this fact is favourable concerning the reduction of the risk of blood atherogenicity in patients with COPD, the identified metabolic situation is the most aggressive risk factor for progression of small steatosis, hepatocyte ischemia and apoptosis, induction of aseptic inflammation, cytolysis and liver fibrosis. An increase in blood pro-inflammatory adipocytokine adipose tissue - leptin and a decrease in blood adiponectin anti-inflammatory action $(p<0,05)$ became a logical explanation for the addition of the inflammatory component to hepatic steatosis in the formation of stetohepatitis on the background of COPD. Considering the fact that COPD promotes several or all of these processes: increasing the degree of IR, intensity of lipolysis in visceral fat depots, as well as in lipocytes of the lung tissue, increasing the intensity of oxidative stress and increased oxidation of lipoproteins carrying TG and cholesterol to the liver with their subsequent deposition, oxidation of lipids of hepatocyte membranes and other tissues, oxidative modification of insulin receptor proteins, leptin, which contributes to a significant disorder of the regulation of lipid metabolism, energy starvation, acceleration of apoptosis and cytolysis, as well as induction of fibrosis, COPD can be considered as a significant risk factor for the progression of NASH against its ground.

\section{Conclusions.}

1. Comorbid COPD in obese and NASH patients is an additional, potent inducer of lipid distress syndrome with significantly higher growth (compared to NASH without lung pathology) in the blood of TGs (2.2 vs. 1.9 times) which form the basis of liver steatosis, total cholesterol (1.5 vs. 1.4 times), LDL cholesterol (1.8 times vs. 1.7), and HDL (1.4 times decrease vs. 1.6 times increase in NASH), atherogenic index (1.2-fold decrease vs. 2.5-fold increase in NASH without lung pathology) ( $\mathrm{p}<0.05)$, accompanied by hyperleptinemia (5.4-fold vs. 4.7), adiponectin deficiency (2.4 times against $1.7)$, correlated with the degree of liver steatosis $(r=0.75-0.79)$, fibrosis index $(r=0.55-0.57)$, cytolytic 
activity $(\mathrm{r}=0.37-0.55)$, cholestatic $(\mathrm{r}=0.52-0.63)$ and mesenchymal-inflammatory syndromes $(\mathrm{r}=0.34$ $0.54)$ and are associated with hyperleptinemia $(r=0.67-0.72)$, hypoadiponectinemia $(r=-0.53-0.57)$, and led to an increase in hepatocyte steatosis index (4.2-fold vs. 3.4 for NASH without lung pathology $(\mathrm{p}<0.05))$.

2. Patients with NASH with accompanying COPD and obesity are characterized by higher levels of insulin in the blood ( 2.9 vs. 2.4 times), postprandial glucose (1.3 vs. 1.2 times), higher insulin resistance (HOMA IR) (2.9 vs. 2.4 times), which correlate with the degree of liver steatosis $(\mathrm{r}=0.56-0.73)$, fibrosis index $(r=0.52-0.65)$, cytolysis activity $(r=0.42-0.53)$, cholestasis $(r=0.34-0.47)$ and mesenchymal inflammation $(\mathrm{r}=0.45-0.61)$ and also depend on the blood content of leptin $(\mathrm{r}=0.69-0.78)$ and adiponectin $(\mathrm{r}=-0.56-0.69)(\mathrm{p}<0.05)$.

\section{References}

Anstee QM, Targher G, Day CP. (2013) Progression of NAFLD to diabetes mellitus, cardiovascular disease or cirrhosis. Nat Rev Gastroenterol Hepatol. 10(6): 330-344. doi: 10.1038/nrgastro.2013.41.

Baltieri L, Chaim EA, Chaim FDM, et al. (2018) Correlation between nonalcoholic fatty liver disease features and levels of adipokines and inflammatory cytokines among morbidly obese individuals. Arq Gastroenterol. 55(3): 247-251. doi: 10.1590/S0004-2803.201800000-62.

Dowman JK. (2011) Systematic review: the diagnosis and staging of non-alcoholic fatty liver disease and non-alcoholic steatohepatitis. Aliment. Pharmacol. Ther. 33(5):525-540.

Elliot JG, Donovan GM, Wang KCW, et al.(2019) Fatty Airways: Implications for Obstructive Disease. Eur Respir J. https://doi.org/10.1183/13993003.00857-2019

Jung DH, Shim JY, Lee HR, et al. (2012) Relationship between non-alcoholic fatty liver disease and pulmonary function. Intern Med J. 42: 541-546.

Lazo M, Hernaez R, Eberhardt MS, et al. (2013) Prevalence of nonalcoholic fatty liver disease in the United States: The Third National Health and Nutrition Examination Survey, 1988-1994. Am J Epidemiol. 178:38-45.

Matthews DR, Kennedy ED, Darbyshire JH, et al. (2007) The UK Diabetes Research Network - an opportunity and a challenge. Diabet Med. 24(1):7-9. DOI: 10.1111/j.1464-5491.2007.02005.x

Mykhailovska NS, Miniailenko LE. (2016) The peculiarities of adiponectin and resistin interrelationships with the components of metabolic syndrome in patients with coronary heart disease and concomitant nonalcoholic fatty liver disease. Zaporizkyi medychnyi zhurnal. 5: 25-30.

Radchenko OM, Pylypiv LI, Zukow V. (2017) Lung function testing according leptin levels in patients with chronic obstructive pulmonary disease. Journal of education, health and sport. 7 (3): 231 -8.

Turer AT, Scherer PE. (2012) Adiponectin: mechanistic insights and clinical implications. Diabetologia. 55(9): 2319-2326. https://doi.org/10.1007/s00125-012-2598-x

Viglino D, Jullian-Desayes I, Minoves M, et al. (2017) Nonalcoholic fatty liver disease in chronic obstructive pulmonary disease. Eur Respir J. 49 (6): doi: 10.1183 / 13993003.01923-2016 